\title{
JMCT Monte Carlo Code with Capability of Integrating Nuclear System Feedback
}

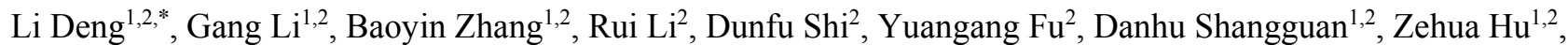 \\ Lingyu Zhang ${ }^{2}$ and Liu Peng ${ }^{2}$ \\ ${ }^{1}$ Institute of Applied Physics and Computational Mathematics (IAPCM), Beijing 100094, China \\ ${ }^{2}$ CAEP Software Centre for High Performance Numerical Simulation(CAEP-SCNS), Beijing 100088, China \\ ${ }^{*}$ Corresponding author
}

\begin{abstract}
JMCT is a general purpose 3D Mont Carlo (MC) neutron-photon-electron and coupled neutron/photon/electron transport code with a continuous energy and multigroup. The program is developed based on the combinatorial geometry parallel infrastructure JCOGIN and with the most functions of general Monte Carlo particle transport code which include the various variance reduction techniques, Doppler broadening onthe-fly (OTF), uniform tally density (UTD), consistent adjoint driven importance sampling (CADIS), fast criticality search of boron concentration, the domain decomposition (DD), the two level parallel computation of MPI and OpenMP, etc. The geometry zones, materials, tallies, depletion zones, memories and period of random number are enough big for simulation of various extreme complicated problems. Also JMCT is hybrid the discrete ordinate $\mathrm{S}_{\mathrm{N}}$ program JSNT to generate source biasing factors and weight window parameters. The CAD modeling and visualized output are equipped. JMCT can provide technology support for reactor physics, criticality safe analysis and shielding design. Especially, JMCT is coupled depletion and thermalhydraulic for simulation of reactor feedback effects, including depletion, thermal feedback. This paper describes the application of the JMCT to the simulation of BEAVRS model. The results are good consistent with MC21, OpenMC and experiment in hot zero power (HZP). Also, we performed the part calculation of hot full
\end{abstract}

power (HFP) in case of 30 axial planes, where the depletion regions exceed 1.528 million and 120 thousand processors to be used in maximum. The capability of high fidelity is shown.

Keywords-monte carlo transport; multi-physics coupled calculation; high fidelity; JMCT; BEAVRS

\section{INTRODUCTION}

JPTS is a high fidelity particle transport system under joint development by IAPCM and CAEP-SCNS for simulation of reactor full-core and radiation shielding (see figure I). This package is developed based on the parallel infrastructure JASMIN [1], JAUMIN [2] and JCOGIN [3], where JASMIN is an adaptive structured mesh infrastructure, JAUMIN is an adaptive unstructured mesh infrastructure and JCOGIN is a parallel combinatorial geometry infrastructure. The infrastructures integrate the mutual and common computational methods and parallel algorithms. It greatly reduces the complicated degree of writing parallel program. In this paper, we mainly introduce the basic functions and key algorithms of JMCT v2.0 [4]. The latest development progress is presented. The test example is chosen the BEAVRS model [5]. The simulated results are presented.

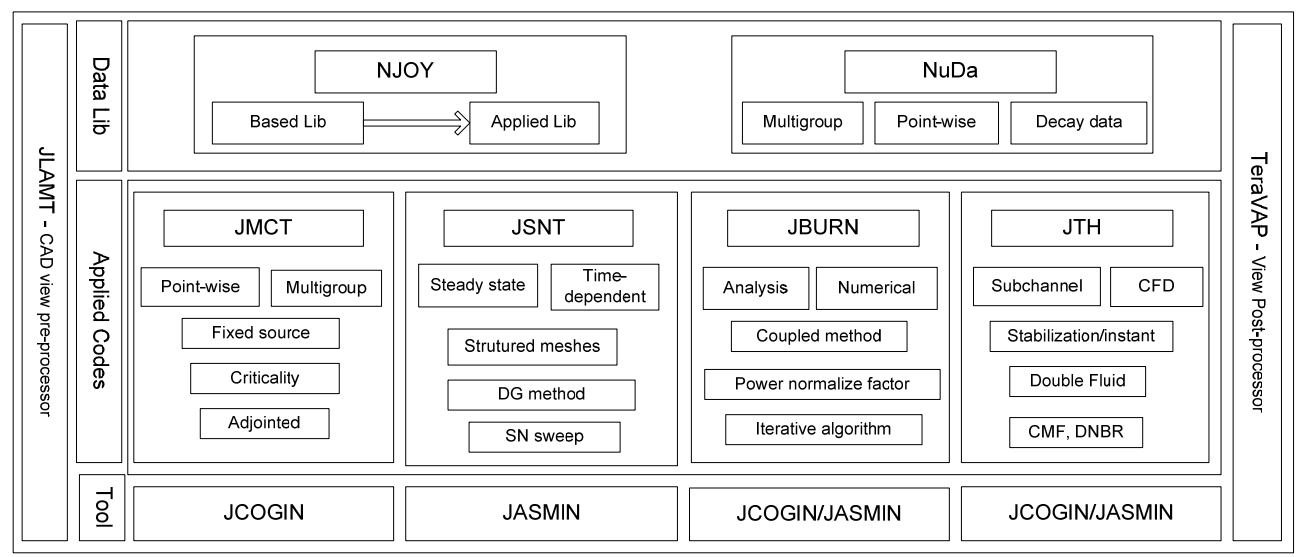

FIGURE I.

THE LOGICAL STRUCTURE OF JPTS PACKAGE

II. Character of JMCT Monte CARlo PARTICle TRANSPORT CODE

The large-scale, multi-functional, three-dimensional neutron-photon-electron transport Monte Carlo program
JMCT can simulate transport problems in any complex geometric systems. The problems of criticality, fixed sources and adjoint are available. JMCT is characterized by strong geometric processing ability, basic geometric bodies include: cube, cylinder, sphere, cone, ellipsoid, torus etc and easy 
extensible. Some special bodies can be customized, such as reactor component, $1 / \mathrm{n}$ pillar, $1 / \mathrm{n}$ sphere, special fuel assembly, etc.. For repetition, JMCT supports repetitive structures with identical geometries and with different materials (MCNP does not support [6]), and is available for tightly coupled calculation of transport and depletion. At present, the material in the geometric cell consists of multiple nucleus including isotopes. The continuous and multigroup energy processing are remained, where multigroup scattering adapts general Gauss quadrature. Energy ranges are $10^{-11}-20$. $\mathrm{MeV}$ for neutrons and $1 \mathrm{eV}-100 \mathrm{GeV}$ for photons and $10 \mathrm{eV}$ $1 \mathrm{GeV}$ for electrons. The cross-section parameters are generated through the NJOY [7], where the based data library is from ENDF/B-VII evaluation libraries [8]. All neutron reaction list in the evaluation library are accounted for. The thermal neutrons are also equipped with corresponding crosssection data, which can be processed with free gas mode or $\mathrm{S}(\alpha, \beta)$ mode. Coherent and incoherent scattering for the photons, and the possibility of fluorescence emission and electron pair generation following photoelectric absorption are taken account.

Program versatility can adapt to simulate a variety of problems. It is equipped with several standard sources and user-defined source. The pre-processing modeling software JLAMT provides interface for user model input including: geometry, material, density, temperature, importance, tally types, source information, sample numbers, wide variety of variance reduction techniques, energy cut-off, weight cut-off, time cut-off, print and writing or reading disk information etc. for restarting. Especial, JMCT tally assorts the global tally and user-specified tally. By default, the global tally is done for all geometric cells according to the track length estimator. The mesh tally is equipped except cell tally. For some specified surfaces tally, it needs to be obtained through body operation.

Domain decomposition (DD) is one of the important algorithms of JMCT. It can simulate those problems which the memory exceeds the limit of single core or node. The huge memory is decomposed on the different processor cores or nodes by DD. In order to ensure the consistency of the series and parallel result in DD and without DD, the random number sequence is elaborately designed except keeping the same Topology relation. In terms of computational methods, the wealth of variance reduction techniques are developed. Especial for deep penetration shielding problems, the coefficients of weight window, importance, mesh window and source biasing are produced by adjoint calculation of $3 \mathrm{D}$ discrete ordinate $\mathrm{S}_{\mathrm{N}}$ transport code JSNT [9] or Monte Carlo code JMCT. Other variance reduction techniques, such as Russian roulette and splitting, implicit capture, forced collisions, source variable biasing, correlated sampling, exponential transformation etc. are included in JMCT. The temperature effect is strictly considered according to the Doppler broadening on the fly. In terms of parallel computing, in addition to the MPI level parallel to the number of particles, the OpenMP secondary parallel function for spatial region decomposition is added. JMCT v2.0 can independently make the transport calculation of multi-types of particles, such as neutron, photon, electron, proton, $\alpha$-particle. The coupled neutron transport, depletion, thermal-hydraulics and fuel performance is developed for reactor lifecycle. The hybrid MC (JMCT) and $\mathrm{S}_{\mathrm{N}}$ (JSNT) has been developed for deeppenetration radiation shielding. The pre-processing and postprocessing have been bundled together with JMCT.

JCOGIN infrastructure integrates the common methods of Monte Carlo particle transport, such as geometry description, Boolean operation, track length calculation, random number generator, DD, parallel computation, sample of arbitrary distribution, volume calculation for arbitrary geometry cell, the advanced computer technology and load balance technologies etc.. It can support the development of various Monte Carlo particle transport programs.

\section{A. JMCT Support}

1) Particle type: neutron, photon, electron and coupled (n, p, e).

2) Mode: forward/adjoint.

3) Problem: fixed source/criticality/activation.

4) Space: $3 \mathrm{D}$ combinatorial geometry.

5) Energy: continuous/multigroup $\mathrm{P}_{\mathrm{N}}(\mathrm{N}=1,3,5)$.

6) Source: standard source/pin-by-pin source/user defined source.

7) Tally: point/surface/cell/mesh.

8) Tally types: $k_{\text {eff }}$, point/surface/volume flux, energy deposition, power, dose rate, detector response and various reactivity etc..

9) Input/Output: CAD modelling and visualization.

10) Parallelization: MPI+OpenMP.

11) Main Algorithms: domain decomposition (DD), uniform tally density (UTD), fast critical search of boron concentration, importance sample, source biasing, mesh tally, mesh windows, implicit capture, Russian roulette and splitting, exponential transform, coupled continuous and multigroup, reactive mechanics about material etc.

12) Temperature: Doppler broadening on-the-fly (OTF) and the cumulative probability method for $S(\alpha, \beta)$.

\section{B. Key Algorithms of JMCT v2.0}

- Domain decomposition of keeping Topology structure [10]

For most Monte Carlo program with DD function, it is very difficult to keep the same result without DD and DD. Such as Mercury code [11], because some cells are divided into two parts by adding surfaces, the calculated results exist difference in DD and without DD. Due to keep the Topology structure, the same results in DD and without DD are obtained for JMCT. The figure II shows the comparison of the pinfluxes in DD and without DD. 


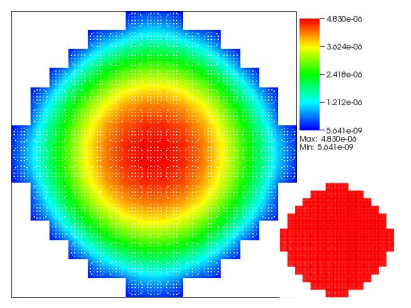

(a)pin flux without DD FIGURE II.

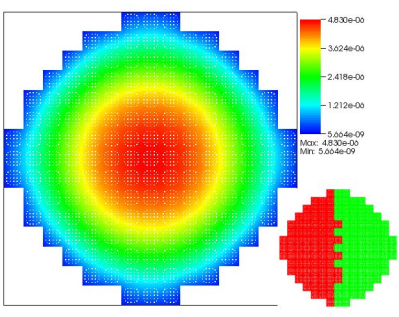

(b)pin flux in $2 \times 1 \times 1 \mathrm{DD}$

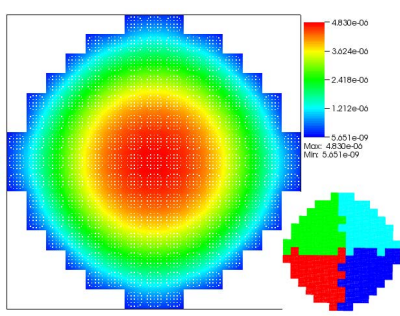

(c)pin flux in $2 \times 2 \times 1 \mathrm{DD}$

RESULT COMPARISON OF DD AND WITHOUT DD CASE.

- High parallel efficiencies in DD

JMCT is designed for massively parallel computing environments and supports both message passing parallelism between compute nodes using OpenMP, as well as shared memory parallelism within nodes using MPI. The parallel efficiency keeps high even in DD case. The figure III(a) shows the parallel efficiency in DD and without DD. The Figure III(b) shows the parallel efficiency of 120 thousand processors for simulation of BEAVRS model [12].

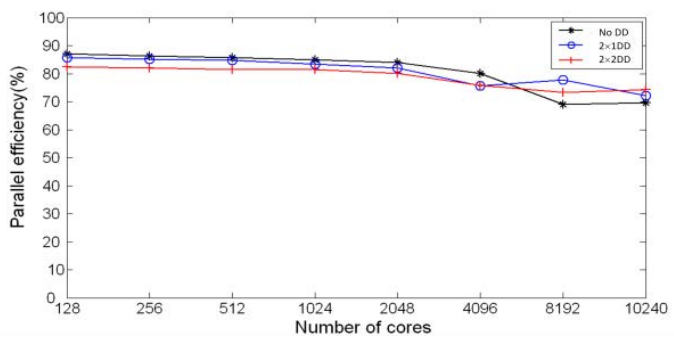

(a) parallel efficiency in DD and without DD

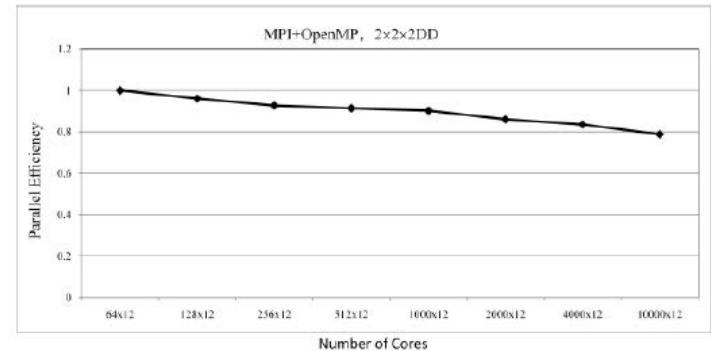

(b) parallel efficiency in 120 thousands CPUs

FIGURE III. PARALLEL EFFICIENCY IN TIANHE-II SUPERCOMPUTER.

- Uniform Tally Density (UTD) for Global Tallying

UTD algorithm is developed based on the following formula

$$
m_{J M C T}^{\text {std }}=w_{\text {in }} \frac{v \Sigma_{f}}{\hat{k}_{e f f} \Sigma_{t}} \frac{v_{k}}{t_{k}}
$$

$$
\begin{aligned}
& \sigma_{x}(v, T)=\sum_{i=1}^{n} \frac{a_{i}}{T^{i / 2}}+\sum_{i=1}^{n} b_{i} T^{i / 2}+C, \\
& T=300 \sim 2000 K, \Delta T=25 K ; x=\text { tot, cap, fis, }
\end{aligned}
$$

It needs to store $2 n+1$ coefficients for each temperature point and energy [14].

- Fast criticality search of soluble boron concentration

The algorithms is based on the neutron balance equation. An equation can be obtained when considering the significant reactions, such as $(n, a),(n, f),(n, \gamma)$ and $(n, 2 n)$ :

$$
\int_{v}\left(\rho_{b} \sigma_{\text {boron }}+\Sigma_{n, \gamma}+\Sigma_{f}+\Omega \cdot \nabla-\Sigma_{n, 2 n}\right) \phi d \vec{r}=\frac{1}{k_{\text {eff }}} \int_{v} F \phi d \vec{r},
$$

where, $\sigma_{\text {boron }}$ is the microscopic cross section of soluble boron, $\Sigma$ are the related macroscopic cross sections, $F$ present the neutron produced by fission. Assuming the ratio of soluble boron density to water density being the same in the whole reactor, the boron concentration can be iterated together with $k_{\text {eff }}$ during the cycles [15].

\section{- Hybrid Monte Carlo and deterministic SN method}

In order to deal with the deep-penetration radiation shielding problem, the adjoint calculation is developed in JMCT and JSNT. Mesh weight windows, importance values and source biasing coefficients are produced by adjoint calculation either $\mathrm{MC}$ or $\mathrm{SN}$. The figure IV shows the calculated flow of coupled Monte Carlo and SN, where the coefficients of Monte Carlo calculation are provided by SN code JSNT [16-17].

- Multi-physics coupled calculation

Tightly coupling of the neutron transport (JMCT), depletion (JBURN) and thermal-hydraulics (JTH) has been developed for simulation of reactor operation. The figure $\mathrm{V}$ shows the calculated flow.

Bigger FOM value is obtained [13].

- Doppler broadening on-the-fly (OTF)

It is based on the following formula 


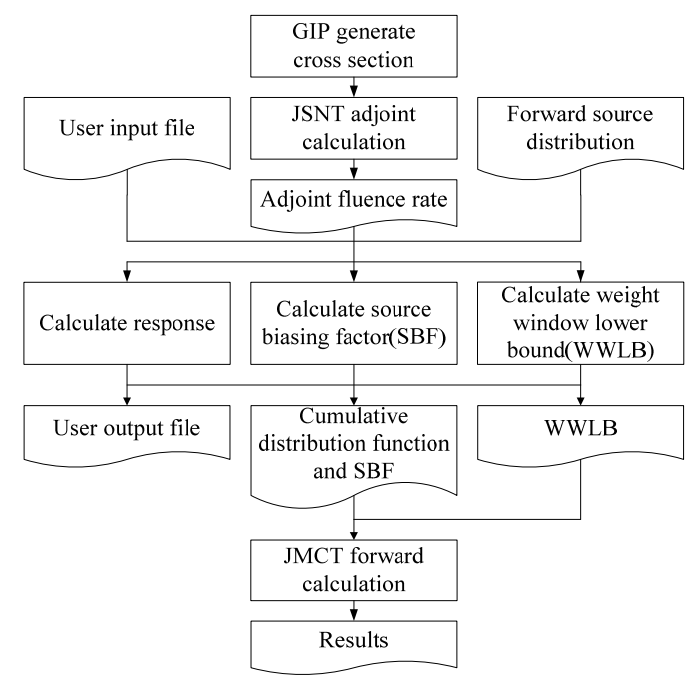

FIGURE IV. FLOW OF THE COUPLED MC/SN.

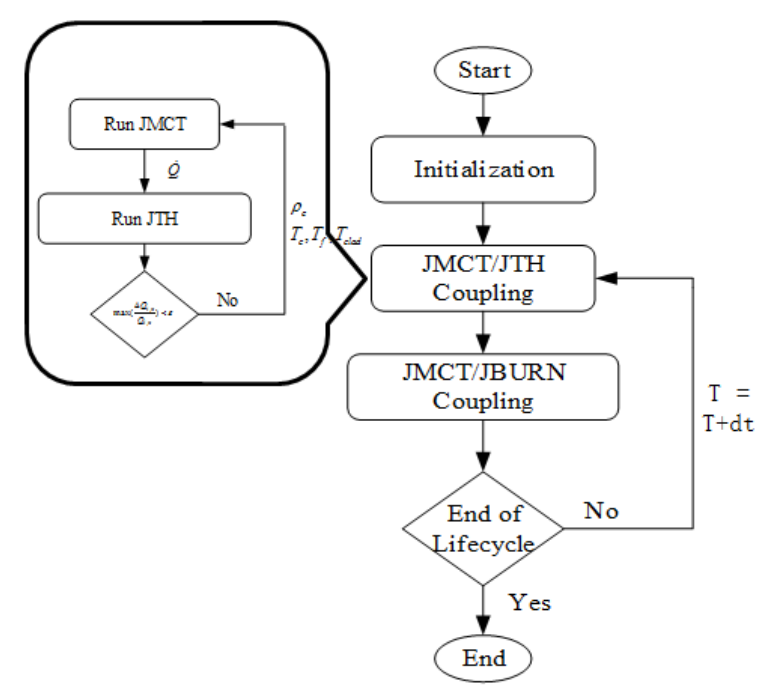

FIGURE V. SCHEMATIC OF THE COUPLED CALCULATION.

\section{NUMERICAL TESTS}

\section{- Simulation of HZP Status}

The BEAVRS model was released by MIT Computational Reactor Physics Group in M\&C2013 conference. It includes detailed specification of operating 4-loop Westinghouse PWR (3411MW), two cycles of measured data, HZP/full power data, fuel loads by assembly as built, three enriched fuels $(1.6 \%, 2.4 \%$ and $3.1 \%$ ). Two cycles of measured data can be used to validate high-fidelity core analysis codes. The part results of MC21 and OpenMC were presented [18-21]. The detailed model is built in the HZP status by pro-processor JLAMT of JMCT. Figure VI(a), (b) and (c) shows the rods, assembles and grids, where 398 layers are in axial direction. Figure VI(d) shows the $2 \times 2 \times 2 \mathrm{DD}$. The tallies do for all pins, the simulation tracks 4 million neutrons /each cycle, 1000 cycles in total and discarding 400 cycles. Figure VII shows the comparison of integrated assembly power between MC21 and JMCT. The maximal difference is $3.17 \%$ in E2 assembly and minimum difference is 0 in E6 assembly. Figure VIII shows the comparison of detectors tallies and the measured data in the instrument tubes. The maximal difference of JMCT result and measured data is $-14.77 \%(-17.05 \%$ for $\mathrm{MC} 21)$ in $\mathrm{B} 13$ assembly. The difference of the minimum power assembly is $5.648 \%$ in L15 assembly. At same time, the comparison of axial detector signals in assemblies B13 and L15 are done (see figure IX). Figure X(a), (b) shows the comparison of radial pin power in the same axial plane (eleventh) between MC21 and JMCT and figure $\mathrm{X}(\mathrm{c})$ shows the standard deviations of pin powers where the standard deviation of $99 \%$ fuel pin powers are less than $1 \%$. The result of JMCT and MC21 have a very good agreement. Table I shows the $k_{\text {eff }}$ comparison of JMCT, OpenMC and MC21 in different location of control rods and boron concentrations. Table II shows the reactivity worth of control rods in $560^{\circ} \mathrm{F}$. Table III shows the comparison of temperature coefficient. Integrated axial power distribution of experiment has slight asymmetry. We guess that it is caused by instrument tubes. Generally, the difference between JMCT and MC21 are small and good agreements.

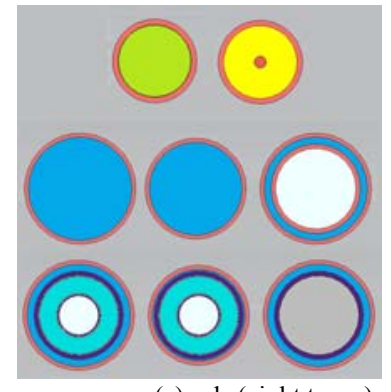

(a)rods (eight types) FIGURE VI.

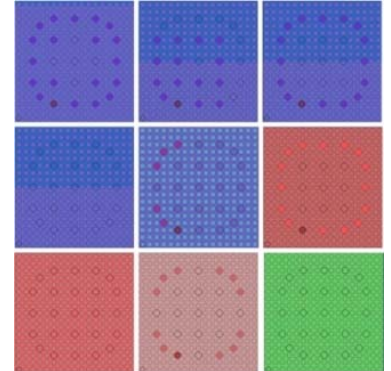

(b)assembles (nine types)

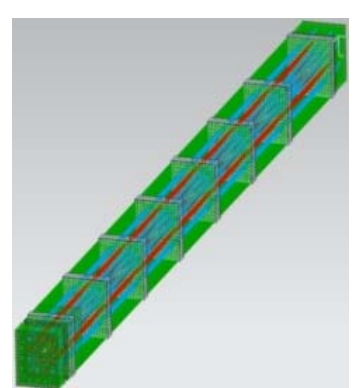

(c)grids

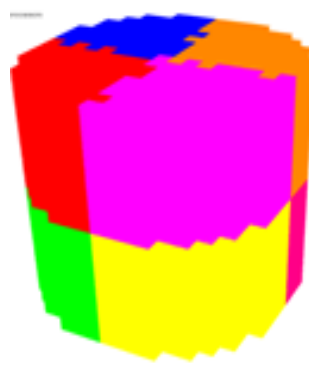

(d) $2 \times 2 \times 2 \mathrm{DD}$

BEAVRS MODELLING BY PRO-PROCESSOR OF JMCT AND DD. 
TABLE I. KEFF COMPARISON IN DIFFERENT CONTROL ROD STATUSES AND BORON CONCENTRATION.

\begin{tabular}{|c|c|c|c|c|}
\hline \multicolumn{2}{|c|}{$\begin{array}{l}\text { Critical Boron Concentrations } \\
(\mathrm{pcm})\end{array}$} & $\begin{array}{c}\text { JMCT } \\
(95 \% \text { confidence })\end{array}$ & $\begin{array}{c}\text { OpenMC } \\
(95 \% \text { confidence })\end{array}$ & $\begin{array}{c}\text { MC21 } \\
(95 \% \text { confidence) }\end{array}$ \\
\hline $\mathrm{ARO}$ & 975 & $1.000479 \pm 0.00003$ & $0.99920 \pm 0.00004$ & $0.9992614 \pm 0.00004$ \\
\hline $\mathrm{D}$ in & 902 & $1.002174 \pm 0.00003$ & $1.00080 \pm 0.00004$ & \\
\hline $\mathrm{C}, \mathrm{D}$ in & 810 & $1.001419 \pm 0.00003$ & $1.00023 \pm 0.00005$ & \\
\hline $\mathrm{A}, \mathrm{B}, \mathrm{C}, \mathrm{D}$ in & 686 & $0.999917 \pm 0.00003$ & $0.99884 \pm 0.00004$ & \\
\hline $\mathrm{A}, \mathrm{B}, \mathrm{C}, \mathrm{D}, \mathrm{SE}, \mathrm{SD}, \mathrm{SC}$ in & 508 & $0.998381 \pm 0.00003$ & $0.99725 \pm 0.00004$ & \\
\hline
\end{tabular}

TABLE II. COMPARISON OF REACTIVITY WORTH OF CONTROL ROD.

\begin{tabular}{|l|c|c|c|c|c|}
\hline \multicolumn{2}{|c|}{ Control Rod Bank Worths (pcm) } & Measure & MC21 & OpenMC & JMCT \\
\hline D & 938.5 & 788 & 773 & $771 \pm 6$ & $770 \pm 6$ \\
C with D in & 856 & 1203 & 1260 & $1234 \pm 7$ & $1258 \pm 6$ \\
B with D,C in & 748 & 1171 & 1172 & $1197 \pm 7$ & $1162 \pm 6$ \\
A with D,C,B in & 748 & 548 & 574 & $556 \pm 6$ & $578 \pm 6$ \\
SE with D,C,B,A in & 597 & 461 & 544 & $501 \pm 6$ & $543 \pm 6$ \\
SD with D,C,B,A,SE in & 597 & 772 & 786 & $844 \pm 6$ & $781 \pm 6$ \\
SC with D,C,B,A,SE,SD in & 597 & 1099 & 1122 & $1049 \pm 6$ & $1107 \pm 6$ \\
\hline
\end{tabular}

Note: 1000 cycles, discarding 400 cycles, 400 million particles per cycle. 200 cores, 5.7 5.8 hours.

TABLE III. TEMPERATURE COEFFICIENT.

\begin{tabular}{|c|c|c|c|c|}
\hline $\begin{array}{c}\text { HZP Critical } \\
\text { Boron Evaluation }\end{array}$ & $\begin{array}{c}\text { Boron } \\
\text { Concentration }\end{array}$ & $\begin{array}{c}\text { Measured } \\
\left(\mathrm{pcm} /{ }^{0} \mathrm{~F}\right)\end{array}$ & $\begin{array}{c}\mathrm{MC} 21 \\
\left(\mathrm{pcm} /{ }^{0} \mathrm{~F}\right)\end{array}$ & $\begin{array}{c}\mathrm{JMCT} \\
\left(\mathrm{pcm} /{ }^{0} \mathrm{~F}\right)\end{array}$ \\
\hline ARO & 975 & -1.75 & -2.7 & -2.21 \\
D in & 902 & -2.75 & -4.3 & -2.99 \\
C,D in & 810 & -8.01 & -9.1 & -6.22 \\
\hline
\end{tabular}

Note: Temperature coefficient under $560^{\circ} \mathrm{F}, \mathrm{T}_{2}=570^{\circ} \mathrm{F}, \mathrm{T}_{1}=550^{\circ} \mathrm{F}$.
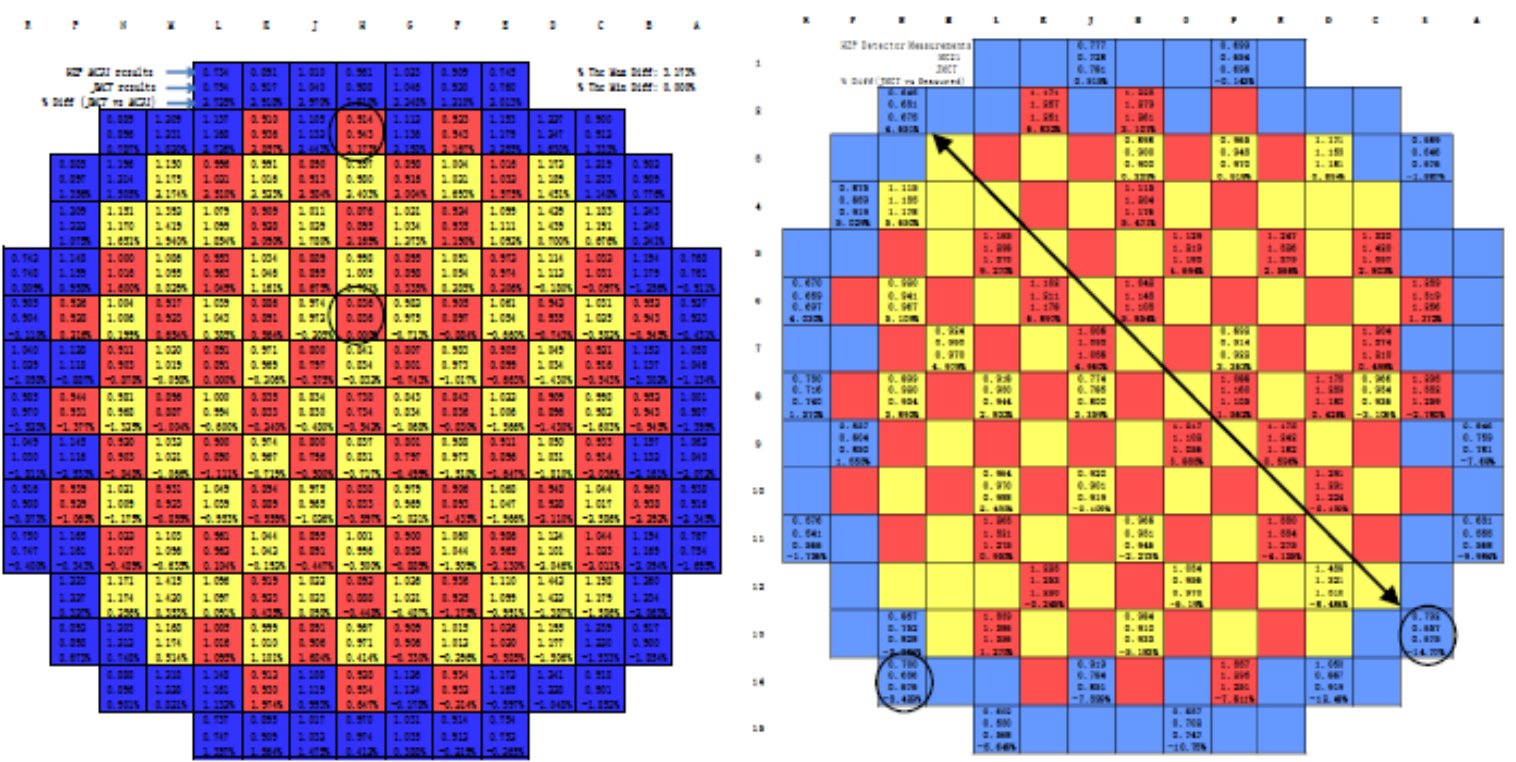

FIGURE VII.

COMPARISON OF PIN POWER DISTRIBUTION BETWEEN MC21 AND JMCT

FIGURE VIII. COMPARISON OF THE DETECTORS DIFFERENCE BETWEEN MC21 AND JMCT 


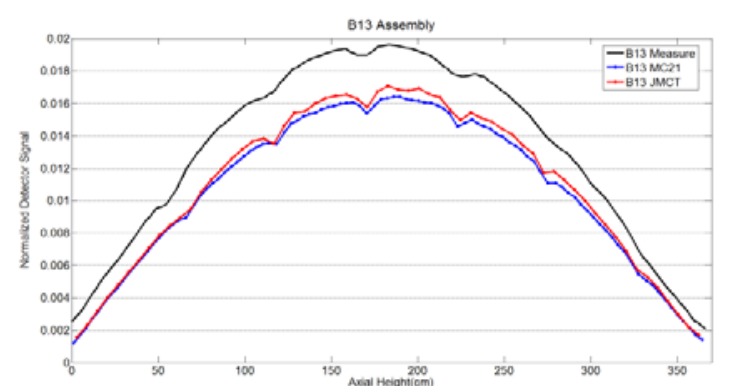

(a)B13 assembly (with maximal difference)

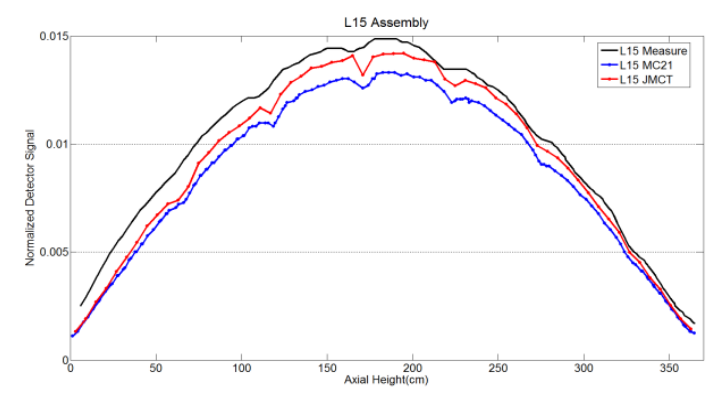

(b)L15 assembly (with minimum power)

FIGURE IX. COMPARISON OF THE AXIAL POWER SHAPE IN B13 AND L15 ASSEMBLEY

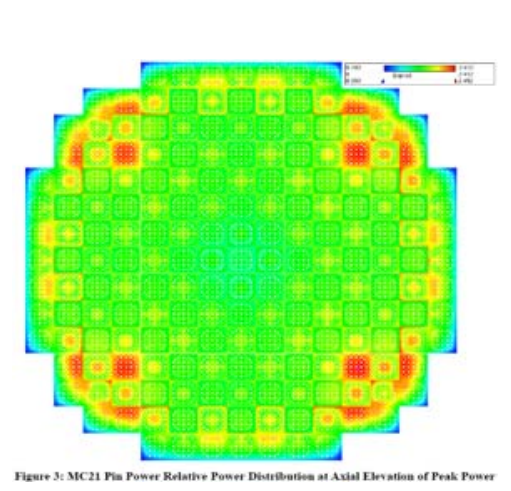

(a) MC21 radial pin power

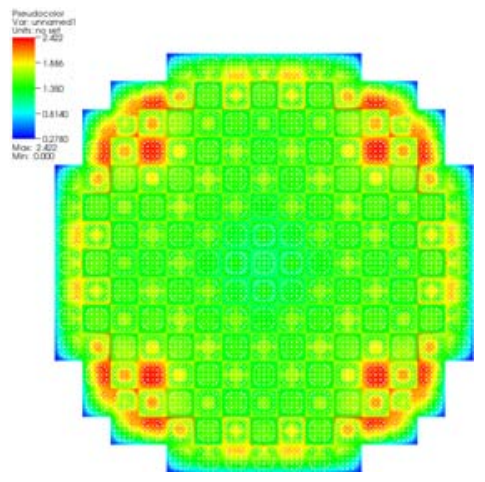

(b) JMCT radial pin power

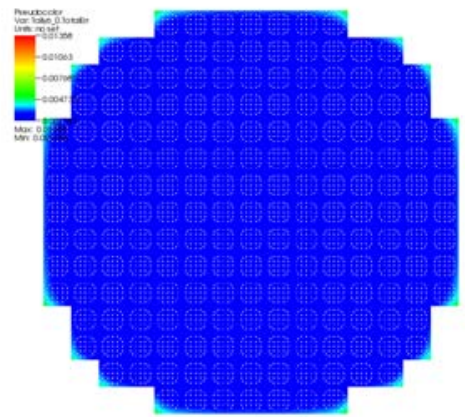

(c) JMCT standard deviation

FIGURE X

COMPARISON OF THE PIN POWER DISTRIBUTION IN RADIAL ELEVENTH PLANE.

\section{- Simulation of HFP Status}

Lifecycle simulation is tried which is coupled neutron transport, depletion and thermal hydraulics. Due to the large memory consume, the simulation runs in 30 axial planes case, where the depletion region is about 1.53 million $(193 \times 264 \times 30=1528560)$. The DD is also decomposed into 8 parts. For each depletion step, 0.5 million neutron histories/each cycle, 200 cycles and 50 discarding cycles, where number of OTF nuclides is 128 and number of depletion nuclides is 3400 . The OTF cross section takes about 4.5 GB memory. The simulation uses MPI and OpenMP for single node of 64GB memory, the memory is more than 50 GB memory for each depletion step. The pin power is convergence after 4 to 5 iterations, where the convergence standard is the ratio of pins power change which satisfies the formula: $\left(Q_{i, n}-Q_{i-1, n}\right) / Q_{i, n}<0.05$. Due to the computational time to consume too big, only the ten depletion steps are simulated. Here only gives the pin power radial distribution in 0 day, 30 days and 48 days (see figure XI). Figure XII shows the pin power distribution in HZP and HFP cases. The difference is obvious.

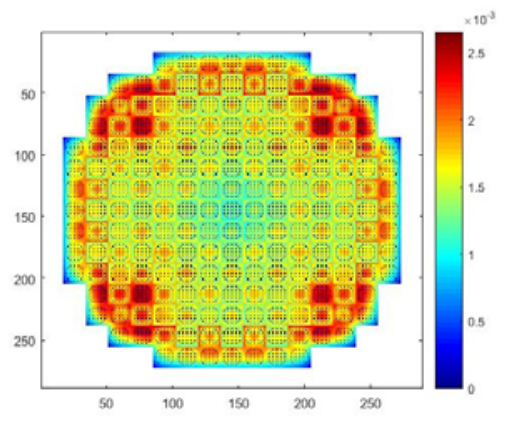

(a) 0day

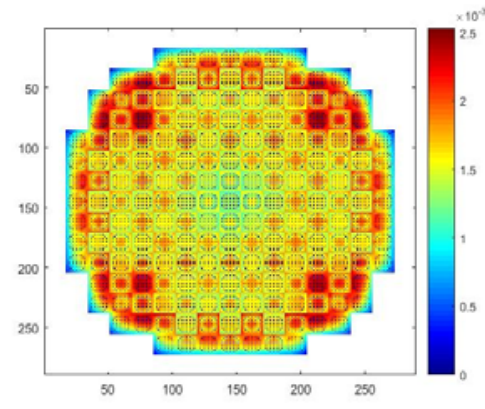

(b)30days

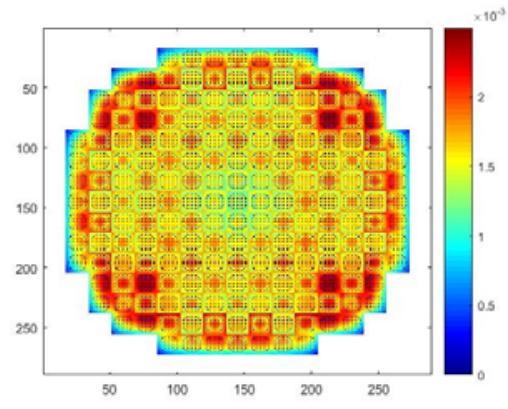

(b) 48 days

FIGURE XI.

RADIAL PIN POWER DISTRIBUTIONS. 


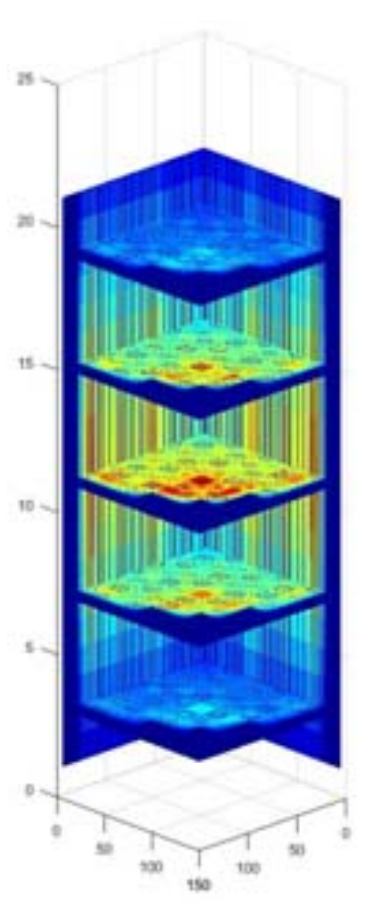

a) HZP

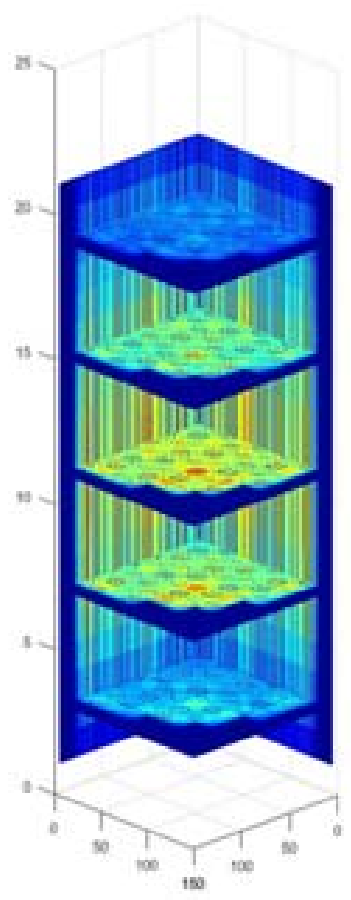

(b)HFP
FIGURE XII. COMPARISON OF PIN POWER DISTRIBUTIONS IN HZP AND HFP STATUS.

\section{SUMMARY}

A general-purpose Monte Carlo particle transport program JMCT has been developed for integrated simulation of nuclear system. It is designed for the simulation of the reactor full-core and radiation shielding. Advanced computer technologies, automatic CAD modelling and visualization make the code with high efficient. JMCTv2.0, the latest version, has with the capability of core analysis.

The future efforts will be forced on enhance of the computing efficiency. The depletion complicating uncertainty quantification and propagation of error need to be considered. Furthermore, it needs to search some new algorithms to reduce the computing fee. Some challenges still face in simulation of the BEAVRS model. JPTS is still in its evolution process toward this goal and all of the algorithms being actively developed at present.

\section{ACKNOWLEDGMENT}

This work was supported by the National Energy Administ ration of China (2015ZX06002008), National Magnetic

Confinement Fusion Energy Research Project (2015GB108 002B), Project (32102010406) and the Technology and Industr y for National Defence (C1520110002).

\section{REFERENCES}

[1] Z. Mo, A. Zhang, X. Cao, et al., "JASMIN: a parallel software infrastructure for scientific computing. Front." Compute. Sci. China, 4(4): 480-488 (2010).

[2] Qingkai Liu, Weibo Zhao, Jie Cheng, et al., "A programming framework for large scale numerical simulation based on unstructured mesh," Proceedings of IEEE-HPSC16, 298-303 (2016).
[3] Baoyin Zhang, Gang Li, Li Deng, et al., "JCOGIN: A PARALLEL PROGRAMMING INFRASTRUCTURE FOR MONTE CARLO PARTICLE TRANSPORT," PHYSOR 2014, Kyoto, Japan, (September 28-October 3, 2014).

[4] Li Deng, Tao Ye, Gang Li et al., "3-D Monte Carlo Neutron-Photon Transport Code JMCT and Its Algorithms," PHYSOR 2014, Kyoto, Japan, (September 28-October 3, 2014).

[5] "MIT Benchmark for Evaluation And Validation of Reactor Simulations," RELEASE rev. 1.0.1, MIT Computational Reactor Physics Group, (July 7, 2013).

[6] R. E. MacFarlane, D. W. Muir, 1994, The NJOY Nuclear Data Processing System-Version 91, LA-12740-1994.

[7] X-5 Monte Carlo team, "MCNP-A General Monte Carlo Code for NParticle Transport Code," version 5 manual, LA-UR-03-1987 (April 24, 2003).

[8] P.F.Rose, Compiler and Editor, "ENDF-201, ENDF/B-VI Summary Documentation," BNL-NCS-17541, Brookhaven National Laboratory (1991).

[9] Tangpei Cheng, Junxia Wei, Guangchun Zhang, et al., "Acceleration of Discrete Ordinates Calculations using Parallel Partial Current Rebalance Algorithm and Algebraic Multigrid Solver." PHYSOR 2016, American Nuclear Society (2016).

[10] G. Li, B. Zhang, L. Deng et al., "Combinatorial Geometry Domain Decomposition Strategies for Monte Carlo Simulations," M\&C 2013, Sun Valley, Idaho, May 5-9, pp.434-443 (2013).

[11] R. Procassini, D. Cullen, G. Greenman, et al., "New capabilities in MERCURY: a modern Monte Carlo particle transport code," M\&C+SNA, Monterey California, (April 15-19, 2007).

[12] Baoyin Zhang, Gang Li, Li Deng et al., "JCOGIN: A parallel Programming Infrastructure for Monte Carlo Particle Transport," PHYSOR 2014, Kyoto, Japan, paper No. 1102407 (September 28-Oct. 3, 2014).

[13] Danhua ShangGuan, Gang Li, Baoyin Zhang, et al., "Uniform Tally Density-Based Strategy for Efficient Global Tallying in Monte Carlo Criticality Calculation," NUCLEAR SCIENCE AND ENGINEERING, 182(4), 555-562 (2016).

[14] Liu Xiong-Guo, Deng Li, Hu Ze-Hua et al., "Study of on-the-fly Doppler broadening in JMCT Program," Acta Phys. Sin., 65(9), 092501-092505 (2016).

[15] R. Li, L. Zhang, D. Shi, L. Deng, "Criticality search of soluble boron iteration in MC code JMCT, Tallying in Monte Carlo Criticality Calculation," Interational Youth Nuclear Congress 2016 (IYNC2016), Hangzhou, China (Sep. 24-30, 2016).

[16] Zheng Zheng, Qiliang Mei, Li Deng, "Study on variance reduction technique based on adjoint Discrete Ordinate method," Annals of Nuclear Energy, 112,374-386 (2018).

[17] Zheng Zheng, Mengqi Wang, Hui Li, Qiliang Mei, Li Deng, "Application of a 3D Discrete Ordinates-Monte Carlo coupling method to deep-penetration shielding calculation," Nuclear Engineering and Design, 326,87-96 (2018).

[18] D.J.Kelly, T.M.Sutton and S.C. Wilson, "MC21 Analysis of the Nuclear Energy Agency Monte Carlo Performance Benchmark Problem," Proc. Advances in Reactor Physics - Linking Research, Industry, and Education (PHYSOR 2012), Knoxville, Tennessee, American Nuclear Society (April 15-20, 2012).

[19] D. P. Griesheimer, et al., "MC21 v.6.0 - A Continuous-Energy Monte Carlo Transport Code with Integrated Reactor Feedback Capabilities," Ann. Nucl. Energy, 82:29-40 (2015).

[20] P.K. Romano, et al., "OpenMC: A state-of-the-art Monte Carlo code for research and development, " Ann. Nucl. Energy, 82:90-97 (2015).

[21] Daniel J. Kelly, Bryan R. Herman, et al., "Analysis of Select BEAVRS PWR Benchmark Cycle 1 Results Using MC21 and OpenMC," PHYSOR 2014, Kyoto, Japan, (September 28-October 3, 2014). 